\title{
AIAA 2000-3869 \\ Bare Electrodynamic Tether Ground Simulations in a Dense, High-Speed Plasma Flow
}

Brian E. Gilchrist

University of Michigan, Ann Arbor, Michigan 48109

Sven G. Bilén

The Pennsylvania State University, University Park, Pennsylvania 16802

Travis A. Patrick and Jonathan L. Van Noord University of Michigan, Ann Arbor, Michigan 48109

36th AIAA/ASME/SAE/ASEE Joint Propulsion Conference and Exhibit 16-19 June 2000 Huntsville, Alabama 


\title{
Bare Electrodynamic Tether Ground Simulations in a Dense, High-Speed Plasma Flow
}

\author{
Brian E. Gilchrist* \\ University of Michigan, Ann Arbor, Michigan 48109 \\ Sven G. Bilén ${ }^{\dagger}$ \\ The Pennsylvania State University, University Park, Pennsylvania 16802 \\ Travis A. Patrick ${ }^{\ddagger}$ and Jonathan L. Van Noord ${ }^{\S}$ \\ University of Michigan, Ann Arbor, Michigan 48109
}

\begin{abstract}
We describe chamber tests of simulated electrodynamic tethers (EDTs) of different geometries operating in a dense, high-speed plasma. The geometries tested were cylindrical, flat-ribbon, and sparse-ribbon or mesh. Several important conclusions can be drawn from the tests as follows: the cylinder collected currents close to what would be predicted via orbital-motion-limited (OML) current collection theory. The tape tether had comparable current levels to a theoretical equal area OML cylinder collector. However, I-V behavior clearly is different at nearest distances ( $\sim 15 \lambda_{D}$ tape width) as compared to furthest test distances $\left(\sim 6 \lambda_{D}\right.$ tape width). The tape tether did better than a theoretical equal mass solid cylinder. A "knee" in the I-V curves can be seen in the tape/mesh data at a potential that is close to the estimated energy of the incoming beam of ions, at least for the closest distances where Debye length is smallest. Below this knee the current increases rapidly as voltage is increased. Above the knee the current increases at a rate one might expect from OML current-collection models depending on the relative width. This likely is an example of high-speed plasma flow effect. Perpendicular tape orientation performed slightly better than parallel.
\end{abstract}

\section{Nomenclature}

$A_{p}$

$d$

I

$k$

$l$

$m_{e}$

$m_{i}$

$n_{e}$

$P$

$q$

$r$

$s$

$t$

$T$

$T_{e}$

$V_{a}$

$v_{t e}$

tape length, $m$

electron mass, $9.109 \times 10^{-31} \mathrm{~kg}$

ion mass, $\mathrm{kg}$

electron plasma density, $\mathrm{m}^{-3}$

power, $W$

charge magnitude, $1.602 \times 10^{-19} \mathrm{C}$

wire radius, $\mathrm{m}$

mesh cylinder spacing, $\mathrm{m}$

tape thickness, $\mathrm{m}$

wire temperature, $\mathrm{K}$

electron temperature, $\mathrm{K}$

accelerating potential, $\mathrm{V}$

electron thermal velocity, $\mathrm{m} / \mathrm{s}$ $w \quad$ tape width, m

$\varepsilon \quad$ spectral emissivity

$\varepsilon_{0} \quad$ free space permittivity, $8.85 \times 10^{-12} \mathrm{~F} / \mathrm{m}$

$\lambda_{D} \quad$ Debye length, $\mathrm{m}$

$\rho \quad$ resistivity, $\Omega \cdot \mathrm{cm}$

$\sigma \quad$ Stefan-Boltzmann constant, $5.67 \times 10^{-8} \mathrm{~W} / \mathrm{m}^{2} \mathrm{~K}^{4}$

* Associate Professor, Electrical Engineering and Space Systems, AIAA Senior Member

${ }^{\dagger}$ Assistant Professor, Communications and Space Sciences Laboratory, AIAA Member

$\ddagger$ Research Assistant, Plasmadynamics and Electric Propulsion Laboratory

$\S$ Research Fellow, Space Physics Research Laboratory, AIAA Member

Copyright (c) 2000 by Brian E. Gilchrist. Published by the American Institute of Aeronatics and Astronatica, Inc. with permiasion.

\section{Introduction}

$\mathrm{T}$ has been proposed that operating in the orbitalImotion-limited (OML) regime is especially beneficial for electron current collection to thin, bare electrodynamic tethers (EDTs) with width dimensions on the order of a Debye length. ${ }^{1}$ This configuration is different than that of Tethered Satellite System missions (TSS-1 and $-1 R$ ), which used a large (with respect to $\lambda_{D}$ spherical collector for electron collection. It is predicted theoretically that a bare tether will be a highly efficient collector of ionospheric electrons (per unit area) when compared to other current collection geometries at equal bias (such as TSS) and it also has been suggested that a combination of technologies may be useful.

As part of NASA's Advanced Space Transportation Program, the Propulsive Small Expendable Deployer System (ProSEDS) mission, ${ }^{2}$ which is to fly in Fall 2000 , will be the first to use the bare-tether concept 
and will demonstrate high current and measurable thrust; the technology is also being considered for other future missions. However, a small, thin cylinder is not necessarily the best tether design when considering other practical factors such as tether lifetime. For example, to increase tether lifetime, a tether based on ribbon-like geometry (e.g., flat and wide) or more sparse structures of equal series resistance may be preferred; hence, these tethers would have dimensions exceeding a Debye length. These new geometries pose several questions that must be addressed before they can be employed. For example, how will the current collection performance change as a function of geometry and Debye length when in an ionospheric plasma at orbital velocities? Does the orientation of the ribbon with respect to the flowing plasma direction have an impact on collection efficiency?

This work describes two sets of vacuum chamber tests that simulated EDTs of different geometries operating in a dense, high-speed plasma. The geometries tested were cylindrical, flat-ribbon, and sparseribbon or mesh* (similar to the so-called Hoytether). For these tests, the 6-m $\times$ 9-m chamber operated by Michigan's Plasmadynamics and Electric Propulsion Laboratory (PEPL) was used along with a PEPL/USAF-designed Hall thruster for the plasma source. These tests were done, in part, to support design efforts for the follow-on mission to ProSEDS called STEP-Airseds, which will demonstrate multiple boost/deboost and inclination-change operations over a period of a year and covering from below $400 \mathrm{~km}$ to above $700 \mathrm{~km}$ altitude.

\section{OML Current Collection}

In the OML regime, the current, $i$, collected by a conductor in non-flowing plasma is given by the equation

$$
i=n_{e} A_{p} q \frac{\sqrt{2}}{\pi} v_{t e}\left(1+\frac{q V}{k T_{e}}\right)^{0.5}
$$

The thermal velocity here is given by $v_{t e}=\sqrt{k T_{e} / m_{e}}$. For high potentials $\left(V \gg k T_{e} / q\right)$, Eqn. 1 reduces to

$$
i=n_{e} A_{p} q \frac{\sqrt{2}}{\pi}\left(\frac{q V}{m_{e}}\right)^{0.5}
$$

We see that for large potentials, the current collected is independent of electron temperature and scales as the square root of the potential.

\section{Experimental Setup \\ Chamber Description and Setup}

PEPL has as its centerpiece the Large Vacuum Test Facility (LVTF), a cylindrical, stainless-steel-clad tank

${ }^{*}$ The cylinder sample had a radius ranging 1-3 Debye lengths (as plasma parameters were varied) whereas the tape/mesh had widths that ranged from 5-19 Debye lengths.

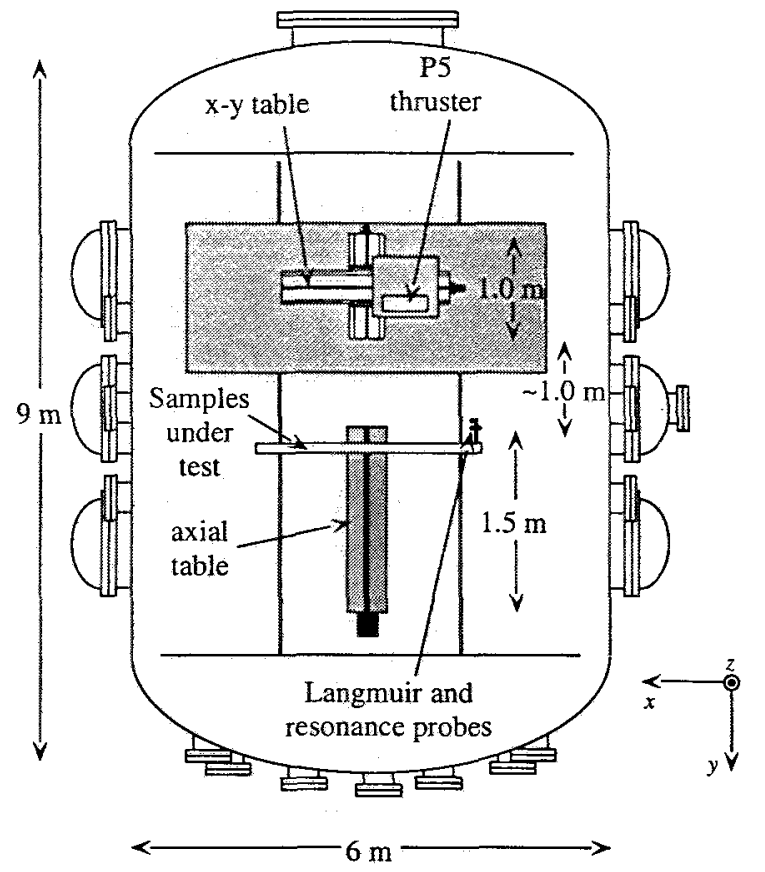

Fig. 1 PEPL LVTF as configured for tests.

that is $9 \mathrm{~m}$ long and $6 \mathrm{~m}$ in diameter. ${ }^{4}$ The facility has four nude cryopumps backed by two $2000 \mathrm{cfm}(56,600$ $l / \mathrm{s})$ blowers and four $400 \mathrm{~cm}(11,300 \mathrm{l} / \mathrm{s})$ mechanical pumps. These pumps give the facility a combined pumping speed of $300,000 \mathrm{l} / \mathrm{s}$ on air and $140,000 \mathrm{l} / \mathrm{s}$ on xenon and provide the ability to reach a high vacuum ( $\sim 10^{-7}$ torr). Fig. 1 is a diagram of the LVTF as it was set up for these tests.

Two positioning tables were used to change the separation distance between the thruster and sample plane and to locate the sample under test (SUT) directly along the thruster's centerline. The thruster was mounted on an $x-y$ table that could move axially $1.0 \mathrm{~m}$ and radially sufficient to cover all samples. The samples were mounted on an aluminum frame that was connected to an axial table that could move $1.5 \mathrm{~m}$ axially (samples are described in more detail below). Combined table movement allowed thrustersample separation distance to change from $\sim 1 \mathrm{~m}$ to $\sim 3 \mathrm{~m}$. Changing separation distance was the primary mechanism for changing the plasma density seen at the sample plane.

\section{Samples}

Three different sample types were constructed for use during these tests: cylindrical, flat-ribbon, and sparse-ribbon or mesh. Tungsten metal was used for all samples to ensure that they would endure the expected high temperatures, which were caused by the collection of high-energy electrons to the samples' surfaces. The rational for and the implications of the use of tungsten are discussed below. During the first 


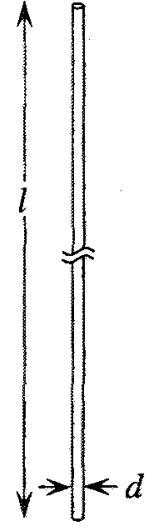

(a)

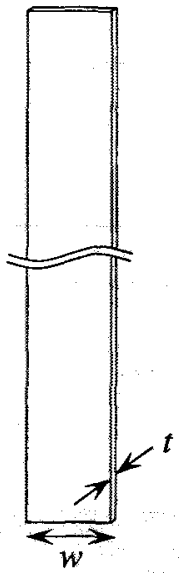

(b)

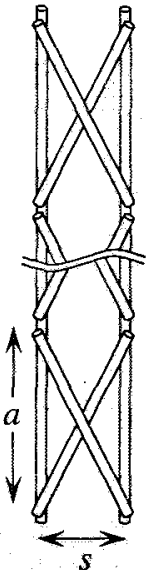

(c)
Fig. 2 Schematics of samples used for both test sets: (a) reference cylinder, (b) tape, (c) mesh.

set of tests, the samples were approximately $10 \mathrm{~cm}$ in length, whereas during the second set of tests, the samples were approximately $30 \mathrm{~cm}$ in length. Two tape samples were used in both test sets, one where the width dimension was perpendicular to the plasma flow, the other with the width dimension parallel to the plasma flow. The thickness of the tape was $t=0.1$ $\mathrm{mm}$. During the first test set, a similar perpendicular/parallel arrangement was used for the mesh samples; however, during the second set only one mesh sample was used and it was primarily perpendicular to the flow. ${ }^{\dagger}$ For the first test set, the mesh samples had a total of five crossing regions, or approximately one crossing region every $2 \mathrm{~cm}$, i.e., $a \approx 2 \mathrm{~cm}$. For the second test set, the mesh sample had 8 total crossings, which means $a \approx 3.5 \mathrm{~cm}$. All mesh samples had a cylinder separation distance, $s$, set to $\sim 2.5 \mathrm{~mm}$. The mesh samples were assembled by spot welding the cross members onto the cylinders where all wire samples were the same diameter as the cylinder sample $(0.28 \mathrm{~mm})$. Sample dimensions were chosen so that appropriate dimensions were on the order of 1-3 Debye lengths. Schematic representation of the samples is given in Fig. 2. Sample descriptions are summarized in Table 1.

All samples were soldered to the center conductor of a bulkhead SHV (safe high voltage) connector; the connector-sample interface was then covered with vacuum epoxy. This sample assembly was then mounted in a piece of stainless-steel flashing, which also functioned as the sample mounting plate. These mounting plates served as localized ground planes and were

tThe mesh sample for the second test set had an approximately quarter spiral curve in it which made part of it perpendicular to the flow and part parallel. These samples were very difficult to construct and this aspect was a result of this sample's construction.

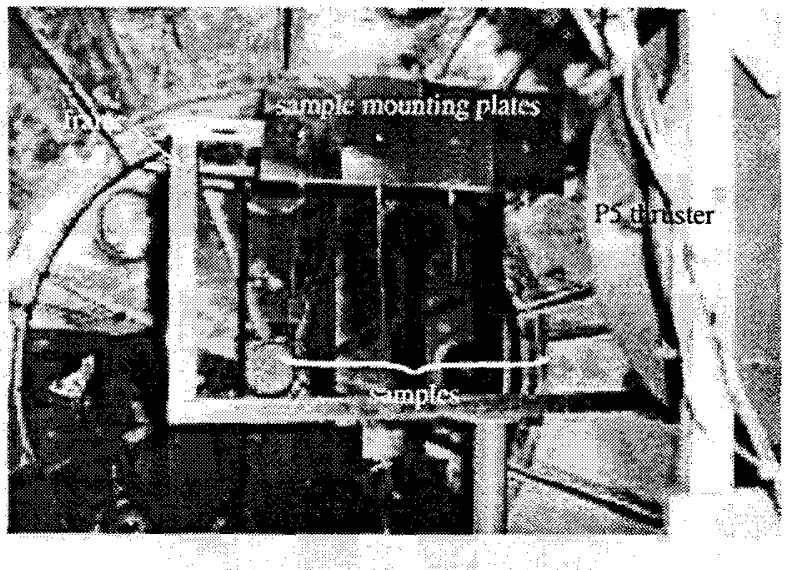

Fig. 3 Picture of the sample support structure for the second test set; structure for the first test set was very similar.

themselves electically connected to the sample support frame. For both test sets there existed a miminum of $10-\mathrm{cm}$ clearance between the samples and the frame. A picture of the setup is shown in Fig. 3 .

\section{Use of Tungsten for Samples}

Due to the energetic electron bombardment of the samples under ligh potentials, the samples would tend to heat up very quickly, and probably past the melting temperature of most metals. Tungsten was therefore used for all samples because it has a melting temperature of $3695 \mathrm{~K}$, which is significantly higher than other metals with low resistivity, such as copper, silver, or gold. Tungsten has a resistivity of $\rho=5.51 \times 10^{-6}$ $\Omega \cdot \mathrm{cm}$, which is within a factor of $2-3$ of these other metals.

By performing the following qualitative analysis, we can determine expected temperatures for the tungsten during current collection. First, we assume the probe is thermally isolated, the surface is equipotential, and finally assume that all electrons collected at the surface (i.e., the electron current to the sample) have been accelerated to the potential of the sample. Then, the power heating the sample is simply $P\left(V_{a}\right)=I\left(V_{a}\right) V_{a}$, where $V_{a}$ is the accelerating potential and the current is a function of $V_{a}$. Fortunately, as the sample's temperature increases, so does its spectral emissivity, $\varepsilon$, which slows down runaway heating slightly. (The electron emission will also increase, but we will ignore this in this analysis.) Using Stefan-Boltzmann's Law, we find the temperature, $T$, of the wire to be

$$
T\left(V_{a}\right)=\left(\frac{P\left(V_{a}\right)}{\sigma A \varepsilon}\right)^{0.25}
$$

From this, we see that $T$ is a function of $V_{a}$. We also note that since $P$ is linearly dependent on $A$ in the OML collection regime, and $A$ is found in the denominator of the right-hand side of Eqn. 3, that surface area 
Table 1 Descriptions of samples used for both test sets.

\begin{tabular}{|c|c|c|c|c|c|c|}
\hline 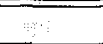 & Sample & 17 & 2 & 3 & 4 & 5 \\
\hline & Description & $\begin{array}{l}\text { Reference } \\
\text { Cylinder }\end{array}$ & $\begin{array}{l}\text { Perpendicular } \\
\text { Tape }\end{array}$ & $\begin{array}{l}\text { Parallel } \\
\text { Tape }\end{array}$ & $\begin{array}{l}\text { Perpendicular } \\
\text { Mesh }\end{array}$ & $\begin{array}{l}\text { Parallel } \\
\text { Mesh }\end{array}$ \\
\hline first & Jength, $l(\mathrm{~cm})$ & 10.0 & 9.4 & 9.2 & 9.8 & 9.5 \\
\hline $\begin{array}{r}\text { test } \\
\text { set }\end{array}$ & $\begin{array}{l}\text { Width, w, or } \\
\text { Drameter, } d(\mathrm{~mm})\end{array}$ & 0.28 & $\begin{array}{l}1.9 \text { (top) to } \\
2.1 \text { (bottom) }\end{array}$ & $\begin{array}{l}2.0 \text { (top) to } \\
2.2 \text { (bottom) }\end{array}$ & $\begin{array}{l}2.5 \text { (top) to } \\
2.3 \text { (bottom) }\end{array}$ & 2.5 \\
\hline second & length, $l(\mathrm{~cm})$ & 29.5 & 29.5 & 29.5 & 27.6 & $\mathrm{n} / \mathrm{a}$ \\
\hline $\begin{array}{l}\text { test } \\
\text { set }\end{array}$ & $\begin{array}{l}\text { Width } w \text {, or } \\
\text { Diameter, } d(\mathrm{~mm})\end{array}$ & 0,28 & $\begin{array}{l}2.0 \text { (top) to } \\
2.4 \text { (bottom) }\end{array}$ & $\begin{array}{l}2.0 \text { (top) to } \\
2.3 \text { (bottom) }\end{array}$ & $\begin{array}{l}2.0 \text { (top) to } \\
2.8 \text { (bottom) }\end{array}$ & $\mathrm{n} / \mathrm{a}$ \\
\hline
\end{tabular}

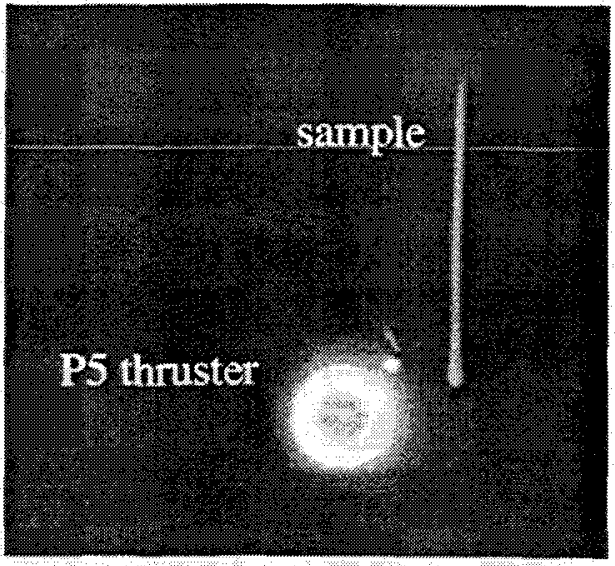

Fig. 4 Picture of a sample glowing due to heating by energetic electron bombardment.

drops out of the equation. What this means is that you are no better or worse of by increasing or decreasing the surface area. Calculations show that at potentials above $\sim 300-400 \mathrm{~V}$ for the plasma densities of these tests, the tungsten is in danger of melting. Hence, a second temperature mitigation method is needed if potentials higher than these values are used. By pulsing voltages at a low duty cycle, the sample is given a chance to cool off before the next pulse of high voltage heats it. The on-time must be short because the heating occurs quickly, on the order of $10 \mathrm{~s}$ of milliseconds. Fig. 4 shows a sample glowing as it is pulsed with $\sim 300 \mathrm{~V}$.

There are some difficulties with using tungsten that should be mentioned as, in pure form, it makes an inappropriate material for EDTs. First, tungsten is very brittle and as such it does not bend well and is not pliable. Second, tungsten is difficult to work and to spot weld to as it very quickly builds up an oxide layer. We worked to overcome these difficulties during these tests because we needed the increased temperature range that tungsten provides.

Thruster Description and Operating Conditions

The PEPL/AFRL "P5" 5-kW-class Hall-effect thruster was used to provide a flowing plasma for these

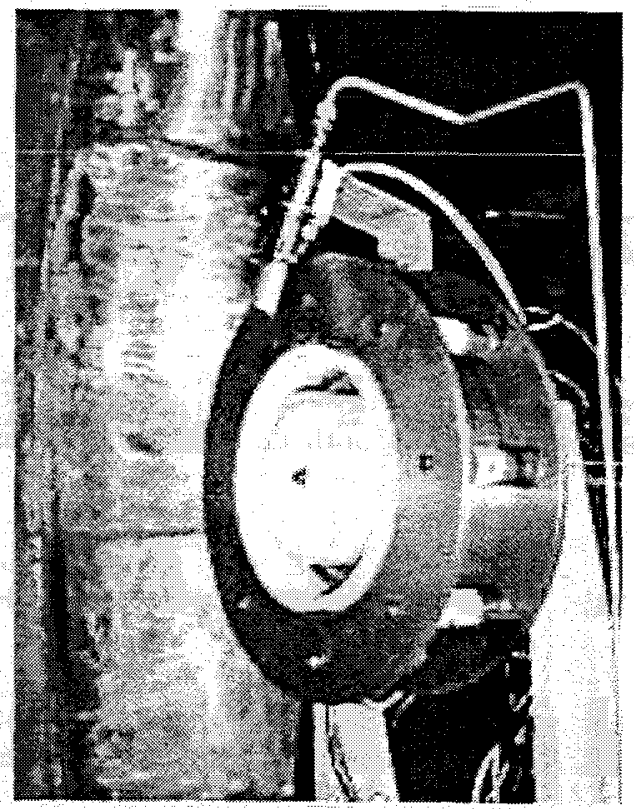

Fig. 5 P5 Thruster.

experiments. A detailed description of the $\mathrm{P} 5$ is given by Haas et al. ${ }^{5}$ and a picture of this thruster is shown in Fig. 5. When operating nominally, the P5 plasma is much too dense for the conditions we needed; hence, for these tests the thruster was set at off-nominal conditions in order to lower the plasma density seen along the thruster's axial direction. The P5 operating conditions for both sets of tests are given in Table 2. For these experiments, the thruster was run on xenon propellant $\left(m_{i}=2.18 \times 10^{-25} \mathrm{~kg}\right)$. An estimate for the average flow velocity is $8-10 \mathrm{~km} / \mathrm{s}$, which yields an ion energy of $\sim 65 \mathrm{eV}$.

We mention at this point several issues regarding the use of the P5 thruster as our plasma source. First, during these tests we did not have sensors available to help us resolve the flow character off axis from the P5. As the P5 is biased off nominal, this may become an issue. That is, the flow may be less directed than when the P5 operates nominally; however, this was partially compensated for by placing each sample in the same location with respect to the thruster at a given distance. 
Table 2 Thruster operating parameters for both test sets.

\begin{tabular}{||l||c|c||}
\hline \hline Parameter & First Test Set & Second Test Set \\
\hline \hline Chamber Pressure (Gauge 1) & $1 \times 10^{-5}$ torr & $6.8 \times 10^{-6}$ torr \\
Chamber Pressure (Gauge 2) & $2 \times 10^{-5}$ torr & $1.5 \times 10^{-5}$ torr \\
Discharge Voltage $\left(V_{d}\right)$ & $100 \mathrm{~V}$ & $100 \mathrm{~V}$ \\
Discharge Current $\left(I_{d}\right)$ & $5.3 \mathrm{~A}$ & $4 \mathrm{~A}$ \\
Inner Magnet Current $\left(I_{\mathrm{im}}\right)$ & $2.99 \mathrm{~A}$ & $1.04 \mathrm{~A}$ \\
Onter Magnet Current $\left(I_{\mathrm{im}}\right)$ & $1.99 \mathrm{~A}$ & $1.03 \mathrm{~A}$ \\
Cathode Voltage $\left(V_{c}\right)$ & $-19.7 \mathrm{~V}$ & $-19.2 \mathrm{~V}$ \\
Heater Voltage $\left(V_{\mathrm{htr}}\right)$ & $7.1 \mathrm{~V}$ & $6.0 \mathrm{~V}$ \\
Anode Flowrate $\left(\dot{m}_{a}\right)$ & $60 \mathrm{sccm}$ & $45 \mathrm{sccm}$ \\
Cathode Flowrate $\left(\dot{m}_{c}\right)$ & $6 \mathrm{sccm}$ & $6 \mathrm{sccm}$ \\
\hline \hline
\end{tabular}

Second, we were not able to measure the population of slow-moving ions. This also may become an issue because of the possibility for charge exchange between and accelerated ion and a slow-moving neutrals. The P5 plasma environment as measured during these tests is presented below.

\section{Data Collection and Measurement System}

\section{Current-Voltage Measurement System}

A schematic of the current-voltage measurement system is given in Fig. 6. The system consisted of a Universal Voltronics BRC 20,000 HV power supply connected to the tether samples by way of a switch box inside the chamber. The HV power supply was controlled via RS -232 by the computer controller running a custom virtual instrument (VI) under LabVIEW. The computer commanded the HV supply to a specified voltage and then quickly back to zero (within $50-100 \mathrm{~ms}$ ) to minimize sample heating. Before returning to zero, the supply's internal voltage sensor reported back the actual voltage obtained. We verified that the supply pulses were long enough such that the voltage had reached a steady-state value. An American Aerospace Controls 835-2-10 current sensor (ammeter) was used to measure current. To increase sensitivity, the HV supply line was looped through the sensor 10 times. Since the output of the ammeter was a voltage signal, an HP $34401 \mathrm{~A}$ voltmeter was used to measure it and, in conjunction with the voltage measurement described above, these values were recorded as voltage-current pairs.

\section{Plasma Parameter Measurements}

Three separate probes of two different types were used to measure the plasma environment. Two Langmuir probes (LPs) were mounted as follows: one parallel to the plasma flow and the other perpendicular to the flow. The LP sweeps were made by a Keithley 2410 source electrometer and collected via a custom VI by a computer controller (Fig. 6). A resonance probe (RP) was also co-located with the LPs. The resonance probe has been used previously to measure plasma densities in the plume of the P5 thruster. ${ }^{6}$ Although still an experimental technique, the $R P$ yields good estimates of density in the far-field region of the P5 plume.

\section{Data}

In order to better understand the data presented here, we present first the plasma environment for both test sets and then the data collected during those tests.

\section{Plasma Environment}

Fig. 7 reports the measured plasma environment as a function of distance for both test sets. By positioning the sample plane closer and farther from the thruster, we were able to obtain approximately a factor-of-10 change in plasma density. There are several qualifications that must be mentioned concerning the determination of the plasma environment. First, LP data were successfully gathered and analyzed for the first round of tests, but RP data were not collected. The LP data from both the horizontal and vertical probes, which require different analyses due to their orientations, were compared and agreed to within a factor of 2. Unfortunately, during the second test set, the LP data could not be trusted. Hence, we utilized RP data for the 187-, 230-, and 305-cm positions. However, we had to estimate the $100-\mathrm{cm}$ position from the OML current fits. Because the RP does not provide $T_{e}$ measurements, we were unable to explicitly measure $T_{e}$ for the second test set, but in comparison with data from the first test set, have set $T_{e}=0.8 \mathrm{eV}$ for all positions. Having said this, we feel that we were able to provide a good description of the plasma environment for all tests.

Fig. 8 shows the calculated Debye lengths for both test sets. Debye length is given by the following equation

$$
\lambda_{D}=\sqrt{\frac{\varepsilon_{0} k T_{e}}{q^{2} n_{e}}} .
$$

The cylindrical sample had a radius $r=d / 2=0.14$ $\mathrm{mm}$, so we can see the Debye lengths obtained spanned a range of $\sim 1-3$ times the cylinder's radius. The tape/mesh width of $\sim 2-2.8 \mathrm{~mm}$ represents from 5-19 times the Debye length. 


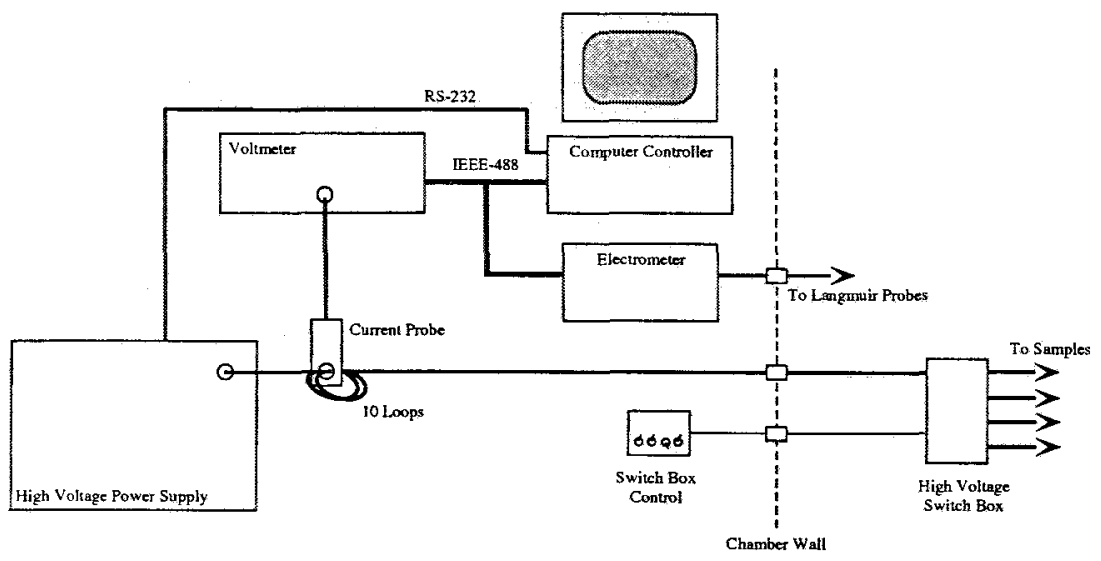

Fig. 6 Schematic of the test equipment setup.

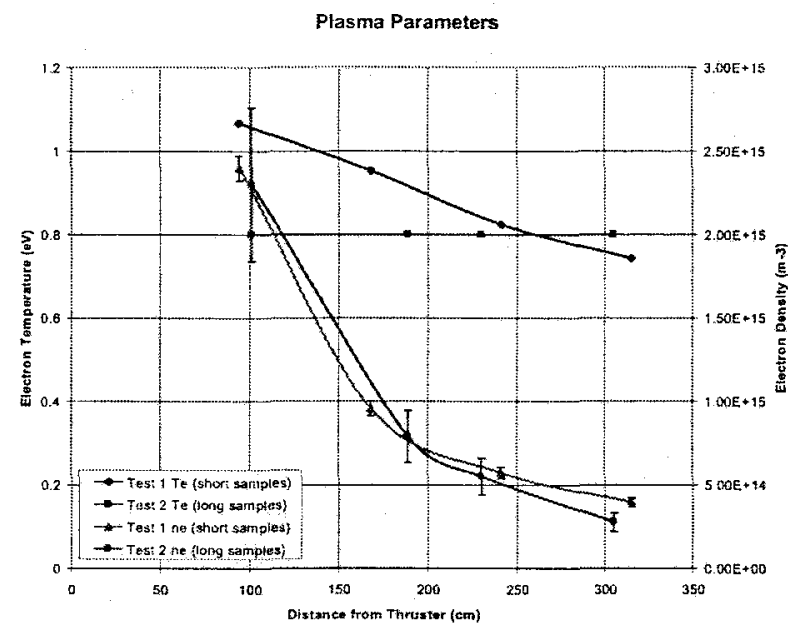

Fig. 7 Plasma parameters measured for both test sets.

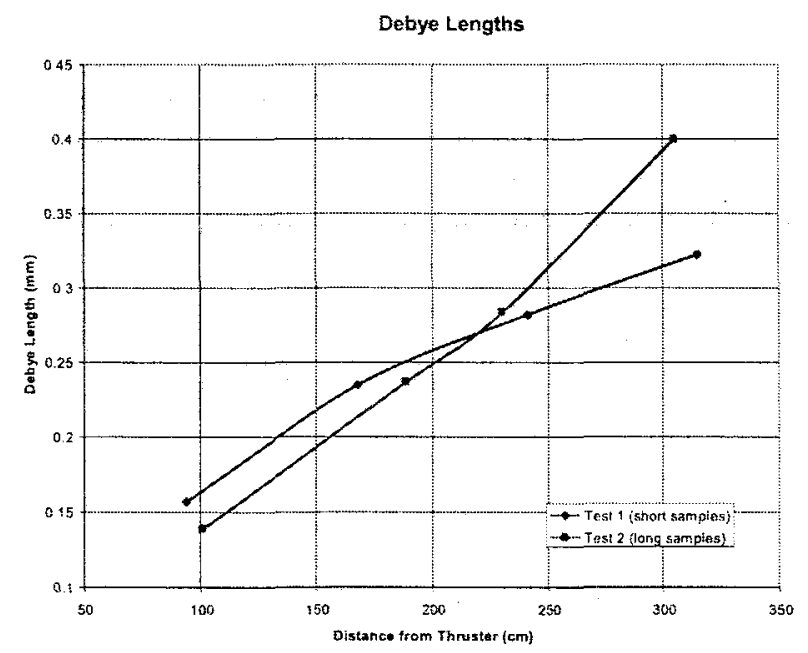

Fig. 8 Debye lengths derived for both test sets.

\section{Current Collection Data}

This first set of figures (Figs. 9a-d) plots the current as a function of voltage for the tape and cylinder geometries from the second test set at separation distances of $305,230,187$, and $100 \mathrm{~cm}$, respectively, which represents lower to higher plasma density (see Fig. 7). They also include the calculated OML currents to equal area equivalent cylinders (the tape equivalent cylinder radius is approximately $7 \mathrm{~mm}$ ). As the separation distance is reduced, the effective width of the probes in terms of $\lambda_{D}$ increases. In general, the calculated model response was near the experimental data. However, the data has important differences in features that will be discussed below. It is also noted that there is some difference between parallel and perpendicular tape orientations.

Fig. 10 provides an example of differences between tests 1 and 2. To compare the differences between the two test runs, given the factor-of-three difference in sample length, the currents obtained in test 1 were multiplied by three before plotting. As seen in the figure, the cylinder samples were approximately the same, however, there is significant difference between the tape tethers. This is thought to be due to sample end-effects and is discussed below.

Fig. 11 provides a comparison of the data during test 2 for the mesh and tape samples (the cylindrical probe results are also added for reference). In this plot, it can be seen that the mesh failed to perform to the same level as the tape even though the tape has a width that is slightly larger than the tape. In the analysis section below it is discussed that this may be an experimental issue, but the inability to collect electrons from the gaps between the wire strands can not be ruled out.

\section{Observations and Analysis}

There are several interesting and intriguing observations that result from the data collected during these 
Comparison of Tape and Cytinder at $305 \mathrm{~cm}$ (Test 2)

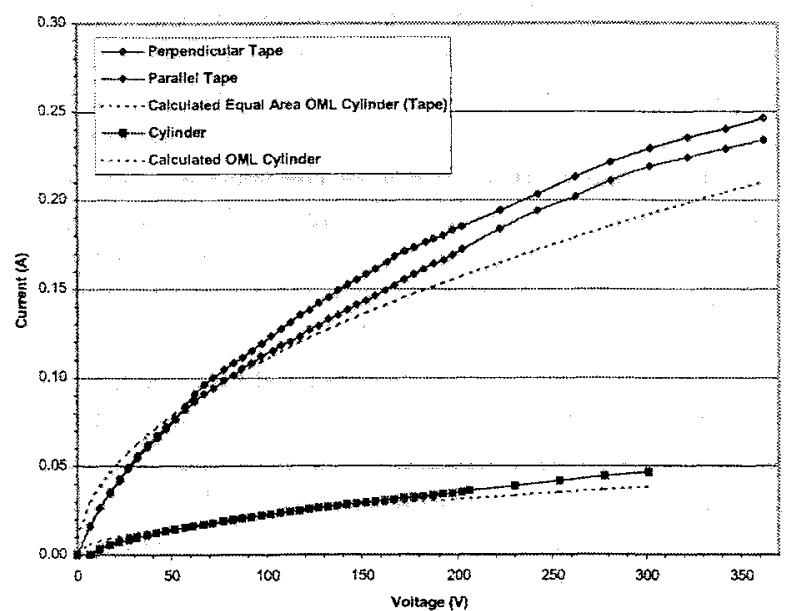

a)

Comparison of Tape and Cylinder at $187 \mathrm{~cm}$ (Test 2)

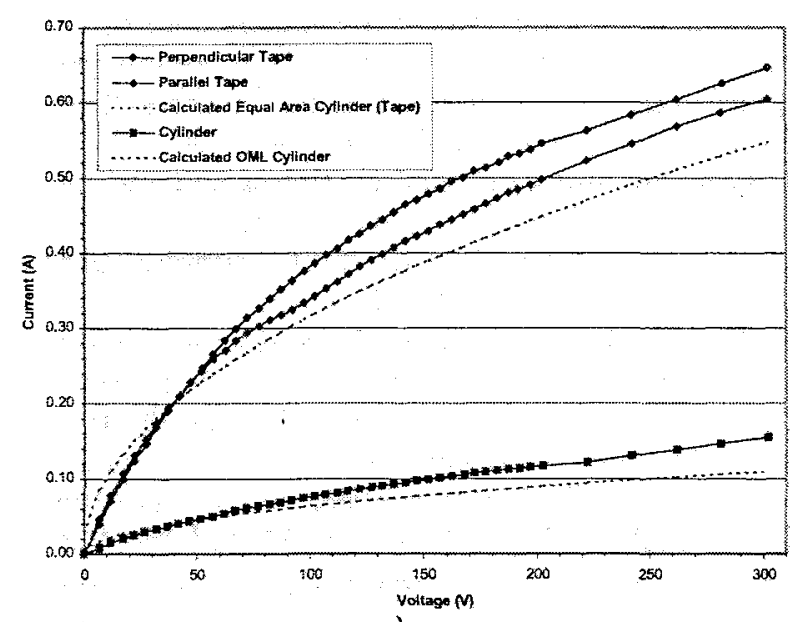

c)
Comparison of Tape and Cylinder at $230 \mathrm{~cm}$ (Test 2)

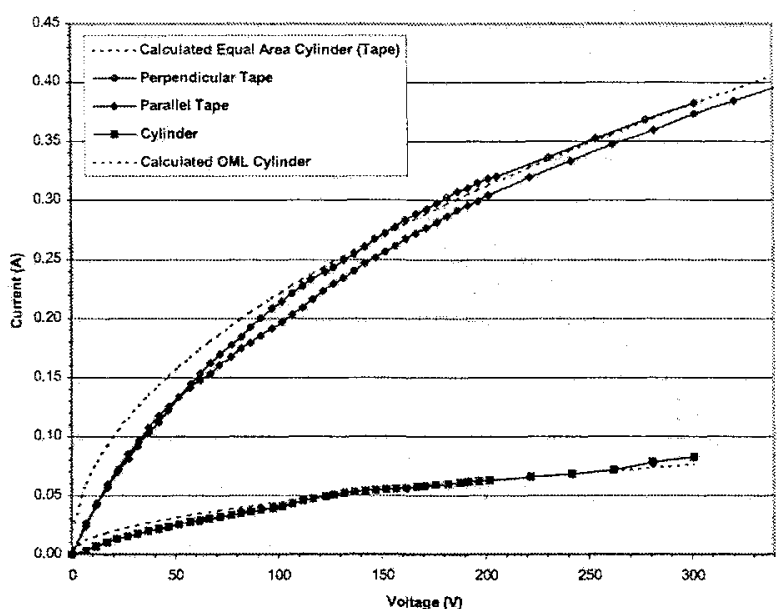

b)

Comparison of Tape and Cylinder at $100 \mathrm{~cm}$ (Test 2)

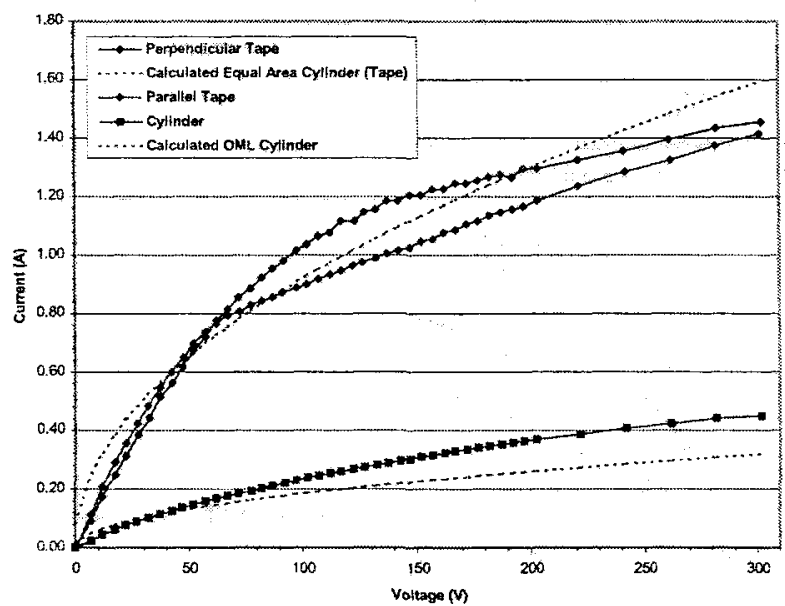

d)

Fig. 9 Current as a function of voltage for the tape and cylinder geometries from the second test set at separation distances of (a) $305 \mathrm{~cm}$, (b) $230 \mathrm{~cm}$, (c) $187 \mathrm{~cm}$, and (d) $100 \mathrm{~cm}$.

tests and presented above. From Fig. 9 it can be seen that there is a small, but definite, difference between parallel and perpendicular orientations of the tape tether. In the figure it can be seen that the perpendicular orientation consistently exceeded the collected current of the parallel orientation and the difference appears to grow at higher densities (shorter Debye lengths). In all cases, collected currents are similar up to $\sim 50-70 \mathrm{~V}$, at which point the collected current deviates for the two orientations. In all but the 100$\mathrm{cm}$ case the difference in current for the orientations appears to reach a constant value that is maintained over the remaining potential range. The $100-\mathrm{cm}$ case appears to show the two curves converging.

We can also interpret this separation between the parallel and perpendicular tape orientations in terms of a "knee" in the $\mathrm{I}-\mathrm{V}$ curves that is particularly pro- nounced for the parallel tape case and is strongest at higher densities. We note that this knee occurs close to the estimated energy of the incoming beam of ions $(\sim 60-70 \mathrm{~V})$. A separate knee is also evident at least for the $100-\mathrm{cm}$ perpendicular tape case at a somewhat higher potential. At potentials below this knee, the current increases rapidly as potential is increased. Above the knee for the 305-, 230-, and 187-cm cases, the current increases at a rate more closely equivalent to OML current collection model. For the $100-\mathrm{cm}$ case, the collected current appears to follow a shallower slope than predicted from OML theory.

We note that the presence of a difference in behavior at potentials below and above the incoming ion energy beam was seen in the TSS $-1 \mathrm{R}^{9}$ and TSS-1 missions. ${ }^{10}$ In these situations, the presence of heating and turbulence could be detected in the plasma over the ion 


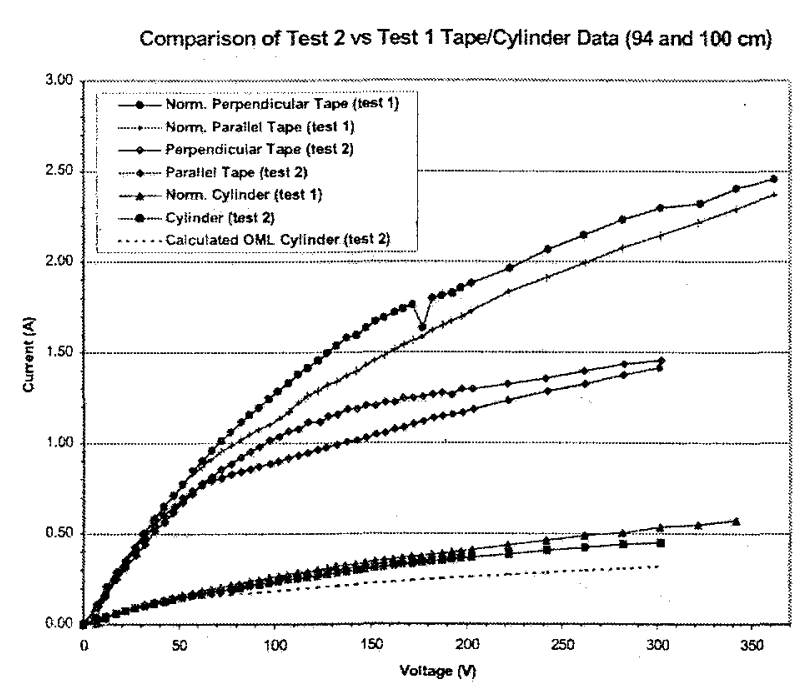

Fig. 10 Comparison of tests 1 and 2.

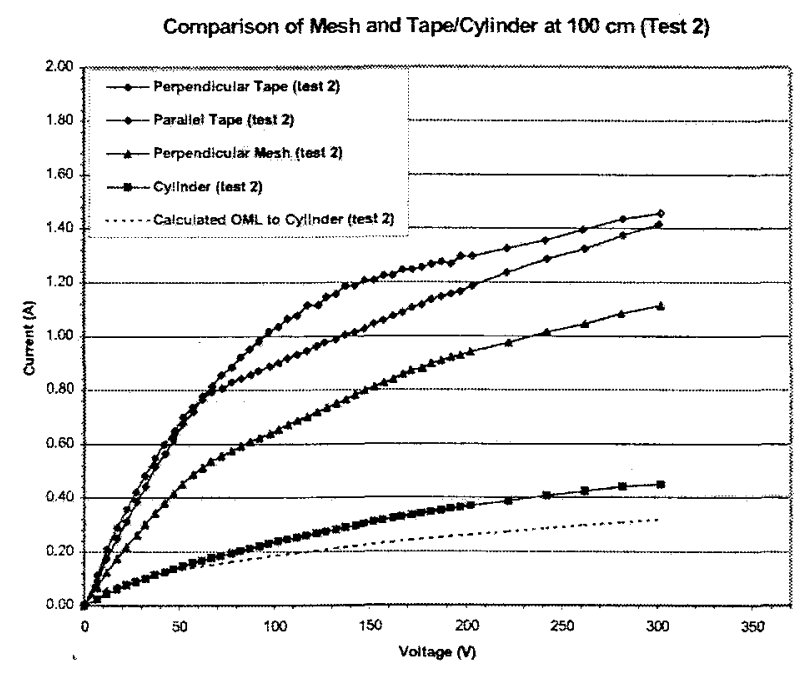

Fig. 11 Comparison of mesh and tape samples for test 2 .

beam energy: Additional measurements will be required to determine if similar effects are present for the tape samples here.

We find it also significant that an equal-area OML cylinder model closely agrees with the prediction of collected current as seen for the tape samples even though the width of the tape is much larger than a Debye length (see Fig. 9). Sanmartín and Estes ${ }^{11}$ considered tape geometries in the OML regime of a stationary, unmagnetized plasma and concluded that there are dimensions beyond a Debye length for conditions that depend on potential and plasma temperatures where a thin tape could satisfy OML collection. Their conclusions for the case appropriate to our experiments would suggest that the tape width could be up to four Debye lengths and still satisfy OML col- lection. Clearly, we have significantly exceeded this dimension here for our moving plasma. An upcoming paper by Estes and Sanmartín ${ }^{12}$ suggests that their earlier threshold predictions are weak, i.e., degradation in performance is not rapid beyond the theoretical limits. This certainly seems to be the case here. Further, the response at $100 \mathrm{~cm}$ where the tape is $\sim 15 \lambda_{D}$ wide and where the current appears to be following a shallower slope may be indicative of exceeding the OML limit. Again, additional experiments will be required to answer this more clearly.

We note in passing that if a theoretical solid cylinder of equal-mass-per-unit-length is considered instead of an equal area cylinder then the experimental results for collected current are about four times larger (not shown). This shows the important practical utility of a thin-tape geometry.

Fig. 10 compares our first and second test runs for the tape and cylinder cases at 94 and $100 \mathrm{~cm}$. Here, the test 1 current has been multiplied by three to crudely compensate for the fact that the samples were three times shorter than the test 2 case. We attribute the difference in the tape results as a result of an end-effect even though the test-1 samples were over $600 \lambda_{D}$ long (end-effects are also multiplied by this same factor of three). In fact, a major reason why the test- 2 run was undertaken was to lengthen the probe samples such that it more completely covered the P5 plume region to minimize end effects. End effects are well known and they are often modeled by an ideal cylinder with an ideal spherical collector at the end, but the effective radius of the spherical collector is found to much wider than probe radius. ${ }^{13}$ It is interesting to note that the cylinder samples did not show as much end effect as seen in the tape samples, which suggests a geometry factor must also be considered. In future experiments, we plan to use appropriate guards at each sample end, although the mechanical complexity for these small probes is considerable.

Fig. 11 compares the test 2 single mesh sample with the tape samples (cylinder is also included for reference). Even though the widths are approximately the same, the mesh under-performs both tape samples. We are concerned that this may be an experimental issue and can not conclusively say that an open mesh of the dimensions used performs less well. Working with tungsten with welded cross members was extremely difficult. Poor electrical connections at the welds is possible. We believe that this work must be repeated using a completely different technique of fabricating the mesh.

\section{Future Tests}

We are planning future experiments to help clarify and expand upon these initial results. First, we will be including a guard to mitigate against end-effects in all samples. We plan on more fully mapping and 
characterizing the plume and its energy distribution. We will be increasing the effective width of the tape samples to approximately $30 \lambda_{D}$. The mesh samples will be manufactured by cutting small holes through a solid tape sample. The density and geometry of these holes will be varied. Finally, we hope to be able to determine if turbulent effects are present in the near plasma as the bias is increased beyond the incoming ion beam energy. Different beam energies will also be used. Magnetic field effects may also be tested but this will require considerable effort for ground-based chamber tests.

\section{Conclusions}

We believe the experimental results to date in highspeed plasma indicate that the tape tether will be a viable option for bare-tether geometries. Widths well above a Debye length can be used, but we are not able to say yet if there is an experimentally determined maximum dimension (e.g., Sanmartín and Estes ${ }^{11}$ ).

The tape sample current collection appears to generally agree with equal-area cylinder OML model. However, there is definitely non-OML character also present in the data, especially for the 100-cm case. Knees in the data appear around the ion beam energy in all cases for the parallel orientation and the perpendicular case at the closest dimensions. It is also possible that at closest dimensions $(100 \mathrm{~cm})$, experimental data is not able to track OML theory at potentials starting around 5 times the ion beam energy. The differences between parallel and perpendicular orientation is also an example of high speed flow effect.

There are questions regarding the mesh performance since it did not perform as well as the solid tape. This is an important question to address because a mesh design (less than 100\% filling) could very well be the best overall design since it has the capability of better survival characteristics in a micrometeoroid environment. We feel strongly that some level of gaps will perform better than an equal-mass solid tape, though more experimental work is required to justify this claim.

The possibility of end-effects even for our longer samples (test 2) does place some question on the overall quantitative assessment of the level of enhancement. In future experiments, a more sophisticated configuration can be used to minimize/eliminate this factor. However, we parenthetically note that adding an end collector may well be a positive enhancement for any bare EDT as our data suggests and suggested by others. ${ }^{\text {? }}$

\section{Acknowledgments}

The authors would like to thank Prof. A. Gallimore for use of the PEPL chamber and the PEPL research group for assistance in performing these experiments. The authors thank Drs. R. Estes, N. Stone, and K. Wright for useful discussions about these results and their interpretation. This work was performed under contract from The Michigan Technic Corporation and NASA-MSFC.

\section{References}

${ }^{1}$ Sanmartin, J. R., Martinez-Snchez, M., and Ahedo, E., "Bare Wire Anodes for Electrodynamic Tethers," $J$. of Prop. and Power, Vol. 9, No. 3, 1993, pp. 353-360.

${ }^{2}$ Johnson, Les, Gilchrist, Brian E., Estes, Robert D., Lorenzini, Enrico, and Ballance, Judy, "Propulsive Small Expendable Deployer System (ProSEDS) Space Experiment," AIAA Paper AIAA-98-4035, 1998.

${ }^{3}$ Forward, R. L., "Failsafe Multistrand Tether Structures for Space Propulsion," AIAA Paper AIAA-92-3214, 1992.

${ }^{4}$ Gallimore, A. D., Kim, S.-W., Foster, J. E., King, L. B., and Gulczinski III, F. S., "Near and Far Field Plume Studies of a One-Kilowatt Arcjet," AIAA J. Prop. Power, Vol. 12, 1996, pp. 105-111.

${ }^{5}$ Haas, James M., Gulczinski III, Frank S., Gallimore, Alec D., Spanjers, Gregory G., and Spores, Ronald A., "Performance Characteristics of a $5 \mathrm{~kW}$ Laboratory Hall Thruster," AIAA Paper AIAA-98-3503, 1998.

${ }^{6}$ Bilén, Sven G., Haas, James M., Gulczinski liI, Frank S., Gallimore, Alec D., and Letoutchaia, Julia N., "ResonanceProbe Measurements of Plasma Densities in Electric-Propulsion Plumes," AIAA Paper AIAA-99-2714, 1999.

${ }^{7}$ Dobrowolny, M., and Vannaroni, F., private communication, 1999.

${ }^{8}$ Stone, N. H., and Bonifazi, C., "The TSS-1R Mission: Overview and Scientific Context," Geophysical Research Letters, Vol. 25, No. 4, 1998, pp. 409-412 (and papers therin).

${ }^{9}$ Thompson, D. C., Bonifazi, C., Gilchrist, B. E., Williams, S. D., Raitt, W. J., Lebreton, J.-P., Burke, W. J., Stone, N. H., and Wright, Jr., K. H., "The Current-Voltage Characteristics of a Large Probe in low Earth Orbit: TSS-1R Results," Geophysical Research Letters, Vol. 25, No. 4, 1998, pp. 413-416.

${ }^{10}$ Katz, I., Melchioni, E., Mandell, M., Oberhardt, M., Thompson, D., Neubert, T., Gilchrist, B., and Bonifazi, C., "Observations of Ionosphere Heating in the TSS-1 Subsatellite Presheath," J. Geophysical Research, Vol. 99, No. A5, 1993, pp. 8961-8969.

${ }^{11}$ Sanmartin, J. R., and Estes, R. D., "The Orbital-MotionLimited Regime of Cylindrical Langmuir Probes," Physics of PJasmas, Vol. 6, No. 1, 1999, pp. 395-405.

${ }^{12}$ Estes, R. D., and Sanmartin, J. R., "Cylindrical Langmuir Probes Beyond the Orbital-Motion-Limited Regime," subrnitted to Physics of Plasmas, 2000.

${ }^{13}$ Johannig, D., Seifert, W., and Best, A. "Analytic Density Correction for Cylindrical Langmuir Probes Showing End Effects, Plasma Physics and Controlled Fusion, Vol. 27, No. 2, 1985, pp. 159-179. 